\title{
Determination of Histamine in Seafood by Hydrophilic Interaction Chromatography/Tandem Mass Spectrometry
}

\author{
Tatsuo Yoshida, ${ }^{*}, * \dagger$ Hirotoshi Hamada, ${ }^{*}$ Hiroshi Murakawa, ${ }^{*}$ Hidekazu Yoshimoto, ${ }^{*}$ \\ Toshiaki ToBIno, ${ }^{*}$ and Kei TODA** \\ *Kumamoto Prefectural Institute of Public Health and Environmental Science, 1240-1 Kurisaki, \\ Uto 869-0425, Japan \\ **Department of Chemistry, Kumamoto University, 2-39-1 Kurokami, Kumamoto 860-8555, Japan
}

\begin{abstract}
A simple method was developed to determine histamine, an important compound in chemical food poisoning, by extraction followed by hydrophilic interaction chromatography-tandem mass spectrometry using a hydrophilic column with sulfobetaine-type zwitterion groups. The quantitation range in seafood products was from 0.4 to $200 \mathrm{mg} \mathrm{kg}^{-1}$ for $5 \mathrm{~g}$ food samples. Quantitative recoveries were obtained with four types of seafood product. These results agreed well with those from the more complex, conventional HPLC method, which requires sample derivatization with dansyl chloride.
\end{abstract}

(Received August 25, 2011; Accepted December 13, 2011; Published February 10, 2012)

\section{Introduction}

Histamine is a compound that causes allergic reactions such as face blushing, headache, urticaria, and fever. ${ }^{1,2}$ As shown in Fig. 1, histamine is formed from histidine contained in food products through decarboxylation by bacteria such as Morganella morganii. Food poisoning with histamine does not occur through the direct effect of bacteria and is classified as chemical food poisoning. Histamine is the most common cause of chemical food poisoning in Japan. There were 89 cases of histamine food poisoning accidents reported, involving 1557 patients, from 1998 to $2008 .^{2}$ The food sources were mostly red flesh fishes, including tuna (33\%), secondary billfish (18\%) and mackerel $(13 \%)$. These species are rich in histidine so greater amounts of histamine tend to be produced from them compared with the amounts from whitefish. There is no regulation of histamine content contained in foods in Japan but the Ministry of Health, Labour and Welfare announced a notification "treatment of sliced fishes from Indonesia" and stated that they order import companies to return polluted products to their origin if the histamine content is detected at more than $200 \mathrm{mg} \mathrm{kg}^{-1}$. Codex standards provide the upper limit of histamine as $100 \mathrm{mg} \mathrm{kg}^{-1}$ for putrefaction standards and $200 \mathrm{mg} \mathrm{kg}^{-1}$ for hygiene standards. Toda et al. indicated that a histamine content lower than $50 \mathrm{mg} \mathrm{kg}^{-1}$ has no effect on human health, but that higher levels than this causes the possibility of food poisoning. ${ }^{2}$

It is mandatory to determine the cause of food poisoning to contain damage in cases where it occurs. Therefore, rapid and reliable methods to qualitatively and quantitatively determine the responsible compounds are required for this purpose. Histamine is a highly polar compound. It cannot be held in a reverse phase chromatography column and its peak tends to be

$\dagger$ To whom correspondence should be addressed.

E-mail: yoshida-t-dp@ pref.kumamoto.lg.jp broad. For this reason, histamine is conventionally derivatized with dansyl chloride ${ }^{3}$ or on-column fluorescence derivatization ${ }^{4}$ before chromatographic analysis or capillary electrophoresis. ${ }^{5}$ These methods require extensive pretreatment before analysis and consume significant time. As a strategy to separate highly polar compounds, on the other hand, hydrophilic interaction chromatography (HILIC) was recently reported. ${ }^{6-10}$ HILIC is a normal phase chromatography method that utilizes a water-organic solvent mixture mobile phase and a more polar stationary phase. Whereas a nonpolar organic solvent is typically used in normal phase chromatography and some hydrophilic compounds cannot be applied because they cannot be dissolved in these solvents, HILIC uses a hydrophilic mobile phase and can be applied to separation of highly polar compounds. Gianotti et al. demonstrated that biogenic amines in cheese could be determined by HILIC coupled with mass spectrometry with atmospheric pressure chemical ionization (APCI). ${ }^{11}$ The polar compound histamine is expected to hold well in an HILIC system with a good peak signal.

In this work, the HILIC method combined with tandem mass spectrometry (MS/MS) with electrospray ionization was investigated for simple measurements of histamine. The HILIC column used was modified with sulfobetaine-type zwitterion groups. We compared the two methods, HILIC-MS/MS method and conventional high performance liquid chromatographyfluorescence detection (HPLC-FL) method, to evaluate the perfomance of the HILIC-MS/MS method.

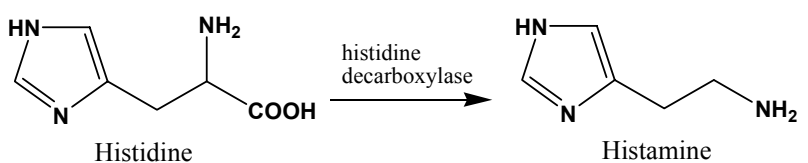

Fig. 1 Chemical structure of histamine formed from histidine. 


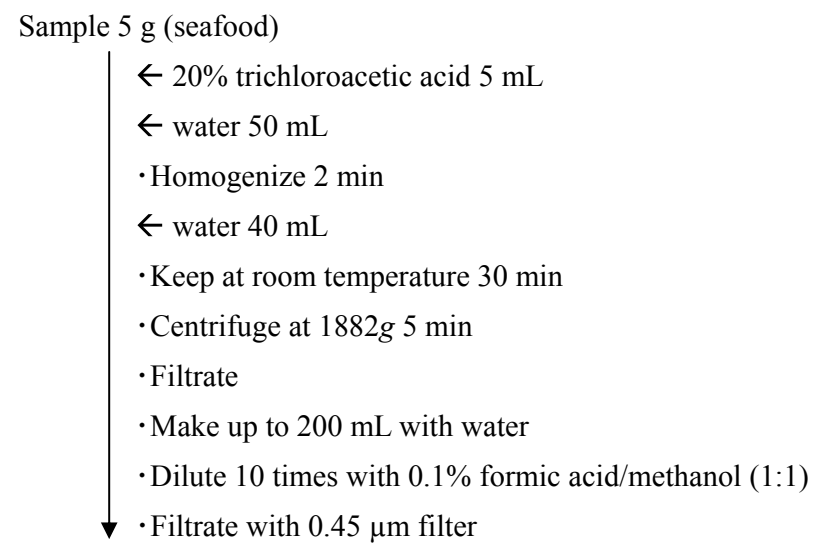

HILIC-MS/MS

Fig. 2 Sample treatment for HILIC-MS/MS.

\section{Experimental}

\section{Reagents}

A stock solution of histamine $\left(1000 \mathrm{mg} \mathrm{L}^{-1}\right)$ dissolved in $0.1 \mathrm{M} \mathrm{HCl}$ was prepared using histamine dihydrochloric acid salt obtained from Wako Pure Chemical Industries, Osaka, Japan. Standard solutions were prepared by diluting the stock solution with a $0.1 \%$ formic acid/methanol mixture $(1: 1)$.

\section{Sample treatment}

Four types of seafood (raw tuna meat, seasoned mackerel, salted mackerel, and open dried horse mackerel), which were confirmed as not containing histamine by the conventional HPLC-FL method, ${ }^{3}$ were homogenized by a food cutter. Histamine standard solutions were added to the homogenized samples to be at the level of 5 or $50 \mathrm{mg} \mathrm{kg}^{-1}$. The histamine-spiked sample was used for spiked recovery tests. Raw tuna, seasoned mackerel, and bonito in brine and oil were used for validation.

The procedure for histamine analysis is shown in Fig. 2. A homogenized seafood sample $(5 \mathrm{~g})$ was further homogenized with $5 \mathrm{~mL}$ of $20 \%$ trichloroacetic acid and $50 \mathrm{~mL}$ of distilled water and then stood for $30 \mathrm{~min}$ after addition of another $40 \mathrm{~mL}$ of distilled water. The sample was centrifuged at $1882 \mathrm{~g}$ for 5 min, then the top clear layer was filtered with a paper filter (GB-140, Advantec, Tokyo, Japan) and diluted with distilled water to a volume of $200 \mathrm{~mL}$ in a volumetric flask. This was then diluted 10 -fold with a $0.1 \%$ formic acid/methanol mixture $(1: 1)$ and filtered with a hydrophobic filter (GL Chromatodisk $25 \mathrm{~N}, 0.45 \mu \mathrm{m}$, GL Sciences, Tokyo, Japan) prior to HILICMS/MS analysis.

\section{HILIC-MS/MS measurement}

The instrument used in this analysis was an HPLC (Waters 2795, Waters, MA) coupled with a tandem mass spectrometer (Quattro Premier, Waters). The separation column was a ZIC-pHILIC obtained from Merck KGaA (Darmstadt, Germany) (4.6 mm i.d. $\times 150 \mathrm{~mm} \mathrm{~L}, 5 \mu \mathrm{m})$, onto which sulfobetainezwitterion type functional groups were covalently bound. Sample injection was $20 \mu \mathrm{L}$ in volume, column temperature was $40^{\circ} \mathrm{C}$, and eluent was distilled water-acetonitrile (ACN)-500 mM ammonium acetate (45:50:5) flowing at the rate of $0.5 \mathrm{~mL} \mathrm{~min}^{-1}$. Each analyte was ionized by electrospray ionization (ESI) in positive mode and quantitative measurement was performed by multiple reaction monitoring (MRM) at the precursor ion $\mathrm{m} / \mathrm{z}$ 117.78 (histamine) and at the secondary ion $\mathrm{m} / \mathrm{z} 94.61$ (amino group removed histamine) produced at a collision energy of $20 \mathrm{eV}$. During the ESI process, the capillary voltage was $3 \mathrm{kV}$, the source temperature was $120^{\circ} \mathrm{C}$, the desolvation temperature and its nitrogen flow rate were $350^{\circ} \mathrm{C}$ and $1000 \mathrm{~L} \mathrm{~h}^{-1}$, respectively; the cone flow rate was $100 \mathrm{~L} \mathrm{~h}^{-1}$.

For comparison of columns, a non-modified silica gel column (Atlantis HILIC, $4.6 \mathrm{~mm}$ i.d. $\times 150 \mathrm{~mm} \mathrm{~L}, 3 \mu \mathrm{m}$, Waters), and columns modified with amide (TSKgel Amide 80, $2.0 \mathrm{~mm}$ i.d. $\times$ $150 \mathrm{~mm} \mathrm{~L}, 5 \mu \mathrm{m}$, Tosoh, Tokyo, Japan), diol (Inertsil HILIC, $4.6 \mathrm{~mm}$ i.d. $\times 150 \mathrm{~mm} \mathrm{~L}, 3 \mu \mathrm{m}$, GL Sciences), and triazole groups (COSMOSIL HILIC, $3.0 \mathrm{~mm}$ i.d. $\times 150 \mathrm{~mm} \mathrm{~L}, 5 \mu \mathrm{m}$, Nacalai Tesque, Kyoto, Japan) were tested.

\section{HPLC-FL measurement}

The conventional analytical method, HPLC-FL adopted as the Japanese standard method, ${ }^{3}$ was used to validate the proposed method. Each sample $(10 \mathrm{~g})$ homogenized with water $(20 \mathrm{~mL})$ was acidified with $10 \mathrm{~mL}$ of $\mathrm{HCl}$ and made up to a volume of $50 \mathrm{~mL}$ with water. After the mixture was left standing for $30 \mathrm{~min}, 1 \mathrm{~mL}$ of the sample solution was placed in a vial. Then $0.5 \mathrm{~mL}$ of 1,8-diaminooctane hydrochloric acid solution as the internal standard, anhydrous sodium sulfate, and a solution of dansyl chloride in acetone were added to form a derivative of the analytes in a $45^{\circ} \mathrm{C}$ water bath for $1 \mathrm{~h}$. After the reaction with $10 \%$ proline was stopped, the derivatives were extracted into $5 \mathrm{~mL}$ of toluene, concentrated and made up to a volume of $1 \mathrm{~mL}$ with ACN. The final sample solution $(10 \mu \mathrm{L})$ was injected into the carrier stream (water: $\mathrm{ACN}=4: 6$ ) flowing at $1.0 \mathrm{~mL} \mathrm{~min}^{-1}$. After the sample passed through the separation column (ODS-3, $3 \mathrm{~mm}$ i.d. $\times 250 \mathrm{~mm} \mathrm{~L}, 5 \mu \mathrm{m}$, GL Sciences) at $40^{\circ} \mathrm{C}$, the fluorescence was monitored at $525 \mathrm{~nm}$ with excitation at $325 \mathrm{~nm}$.

\section{Results and Discussion}

\section{Investigation of analytical conditions}

Water plays an important role in HILIC separation, where a water-rich phase is formed in the stationary phase. ${ }^{9}$ Separation of analytes is performed by partition between the hydrophilic stationary phase and the less hydrophilic mobile phase; the HILIC system tends to be affected by water supplied from the mobile phase. Hence, the retention time and analytical peak area are not stable if the stabilization during equilibration of the column is not sufficient. Therefore, an isocratic condition was adopted to maintain the equilibrium of the stationary phase with the water-ACN mixture.

There are several types of HILIC stationary phases, including non-modified silica gels and those modified with amide, zwitterion, diol, and triazole groups. We tested these columns to select the optimal one for our purpose. Large tailing of the peak was observed with amide- and diol-modified columns. The linearity of the calibration curve was poor with non-modified silica gel $\left(R^{2}=0.832\right)$, amide $\left(R^{2}=0.897\right)$, diol $\left(R^{2}=0.794\right)$ and triazole $\left(R^{2}=0.868\right)$ columns, and separation from the sample matrix was difficult with the triazole column. The best chromatogram was obtained with the HILIC column modified with sulfobetaine zwitterion groups, as shown in Fig. 3. Linearity was sufficient $\left(R^{2}>0.999\right)$ for sample solutions ranging from 1 to $500 \mu \mathrm{g} \mathrm{L}^{-1}$. This stationary phase involves not only hydrophilic interaction but also electrostatic interaction. The number of plates was estimated to be 2566 from the chromatogram. Thus, the column modified with the sulfobetaine 

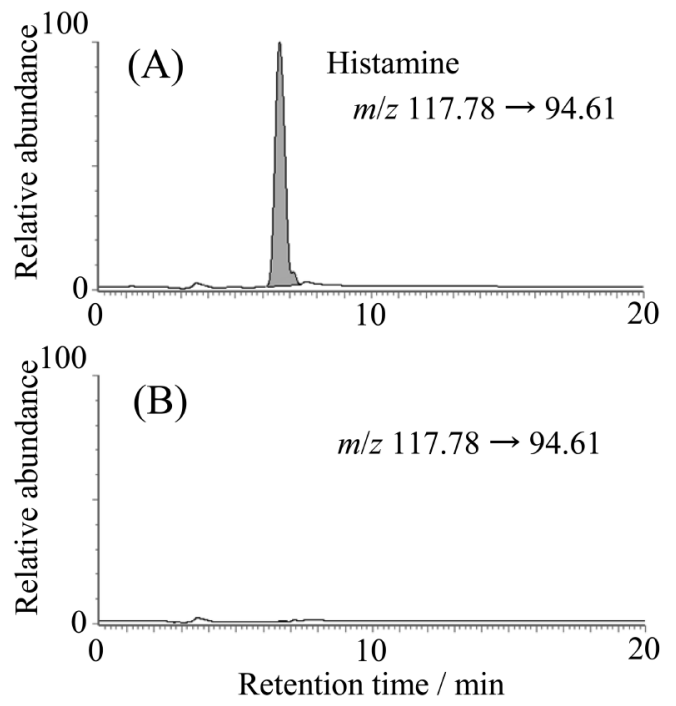

Fig. 3 Chromatograms obtained for extracts from tuna spiked with $50 \mathrm{mg} \mathrm{kg}^{-1}$ histamine (A) and raw tuna (B).

zwitterion was used for further investigation.

We changed the ACN content from 20 to $80 \%$ and found that the best peak shape was obtained with $50 \%$ ACN eluent. Ammonium acetate $(0-25 \mathrm{mM})$ was added to the eluent to stabilize the hydrate layer. The peak shape was improved by increasing the ammonium acetate content; the best peak shape was obtained at $25 \mathrm{mM}$ ammonium acetate. Thus, $25 \mathrm{mM}$ ammonium acetate in $50 \%$ ACN aqueous solution was adopted as the eluent.

For the detection of histamine, positive mode ESI was adopted. Ionization conditions were automatically optimized using the mass analyzer by injecting $1 \mathrm{mg} \mathrm{L}^{-1}$ histamine, introduced directly to the mass analyzer without a separation column. The optimized conditions comprised capillary voltage $3 \mathrm{kV}$, source temperature $120^{\circ} \mathrm{C}$, desolvation temperature $350^{\circ} \mathrm{C}$, cone gas flow $100 \mathrm{~L} \mathrm{~h}^{-1}$ and desolvation gas flow $1000 \mathrm{~L} \mathrm{~h}^{-1}$. Histamine could be ionized to detect with the ESI, whereas Gianotti et al. utilized APCI. ${ }^{11}$ ESI is more popular for ionization in LC-MS/MS analysis and stable signals are usually obtained, with better repeatability.

\section{Performance of HILIC-MS/MS for histamine analysis}

The performance of the proposal method was examined under the optimized conditions; good linearity was obtained in the range from 1 to $500 \mu \mathrm{g} \mathrm{L}^{-1}$ of matrix-matched standard solutions.

$$
\text { Peak area }=2723 \text { histamine }\left(\mu \mathrm{g} \mathrm{L}^{-1}\right)+484, \quad R^{2}=0.999
$$

A typical chromatogram is shown in Fig. 3. By the HILIC-MS/MS method, some sample solution can be introduced by the pretreatment of only dilution without purification because of the high sensitivity and high selectivity of the detection. No interference peak from any of the diverse compounds present was observed and a single clear peak was observed. The lowest concentration of the standard solution used for calibration was $1 \mu \mathrm{g} \mathrm{L}^{-1}$; this corresponded to a $0.4 \mathrm{mg} \mathrm{kg}^{-1}$ seafood sample concentration. For this standard solution, the signal-to-noise ratio of the peak was 80 against the stable area of the baseline; the limits of detection (LODs) estimated from this data were $0.038 \mu \mathrm{g} \mathrm{L}^{-1}$ and $0.015 \mathrm{mg} \mathrm{kg}^{-1}$. The limit of quantification
Table 1 Recoveries of histamine from seafood samples

\begin{tabular}{lclc}
\hline \multicolumn{1}{c}{ Sample } & $\begin{array}{c}\text { Concentration/ } \\
\mathrm{mg} \mathrm{kg}^{-1}\end{array}$ & \multicolumn{2}{c}{$\begin{array}{c}\text { Recovery RSD, \% } \\
(n=6)\end{array}$} \\
\hline Tuna & 5 & 103.5 & 1.5 \\
& 50 & 102.0 & 3.6 \\
Seasoned mackerel & 5 & 103.3 & 2.5 \\
& 50 & 102.6 & 3.4 \\
Salted mackerel & 5 & 103.9 & 1.6 \\
& 50 & 102.0 & 2.5 \\
Open dried horse & 5 & 103.5 & 1.5 \\
mackerel & 50 & 101.5 & 1.5 \\
\hline
\end{tabular}

Table 2 Histamine found in seafood samples by HILIC-MS/MS and conventional HPLC-FL

\begin{tabular}{lrrc}
\hline \multirow{2}{*}{ Sample } & \multicolumn{3}{c}{ Histamine found/mg kg-1a } \\
\cline { 2 - 4 } & HILIC-MS/MS & HPLC-FL & Ratio, \% \\
\hline Bonito in brine and oil & $65.9 \pm 0.8$ & $68.6 \pm 1.2$ & 96.1 \\
Tuna & $25.1 \pm 0.7$ & $25.9 \pm 2.9$ & 96.9 \\
Seasoned mackerel & $8.5 \pm 0.1$ & $8.6 \pm 1.0$ & 98.8 \\
\hline
\end{tabular}

a. Data shown were obtained by measurements repeated in triplicate. b. Ratios are HILIC-MS/MS data to HPLC-FL data.

was $0.13 \mu \mathrm{g} \mathrm{L}^{-1}\left(0.052 \mathrm{mg} \mathrm{kg}^{-1}\right)$. If there is no matrix in the HILIC sample, the noise ratio for $1 \mu \mathrm{g} \mathrm{L}^{-1}$ was 92 ; LOD and LOQ were 0.033 and $0.11 \mu \mathrm{g} \mathrm{L}^{-1}$. Even though the pretreatment was much simpler than that for the conventional HPLC-FL method, the LOD was superior to that obtained by HPLC-FL ( $25 \mathrm{ng}$ in $10 \mu \mathrm{L}$, i.e. $2.5 \mathrm{mg} \mathrm{L}^{-1}$ ). ${ }^{3}$ The measurable range of histamine was from 0.4 to $200 \mathrm{mg} \mathrm{L}^{-1}$. The lowest concentration of the standard was less than $1 / 100$ of the allowable level of food poisoning $\left(50 \mathrm{mg} \mathrm{kg}^{-1}\right)$. Thus, this method has sufficient sensitivity to detect histamine for food safety analysis.

\section{Measurement of histamine contained in seafood samples}

Recoveries $(n=6)$ were examined for four types of seafood samples. For all samples spiked with 5 or $50 \mathrm{mg} \mathrm{kg}^{-1}$, good chromatograms were obtained, as shown in Fig. 3. Results are shown in Table 1. Recoveries obtained for $5 \mathrm{mg} \mathrm{kg}^{-1}$ spiked samples were from 103.3 to $103.9 \%$ with relative standard deviations 1.5 to $2.5 \%$. In case at $50 \mathrm{mg} \mathrm{kg}^{-1}$, they were 101.5 to $102.6 \%$ with 1.5 to $3.6 \%$. No variation of recovery was observed between different samples and good recoveries of histamine were obtained for all seafood samples. Inter-day RSD for six days was $2.2 \%$ for the $50 \mathrm{mg} \mathrm{kg}^{-1}$ spiked tuna meat.

We attempted to detect histamine in seafood samples and could find histamine in three samples from 19 samples examined. Histamine in the same samples was measured also by HPLC-FL, as mentioned in the experimental section. Results are shown in Table 2. Histamine content determined in bonito preprocessed with brine and oil using HILIC-MS/MS and HPLC-FL were 65.9 and $68.6 \mathrm{mg} \mathrm{kg}^{-1}$, respectively. For raw tuna meat, those corresponding contents were 25.1 and $25.9 \mathrm{mg} \mathrm{kg}^{-1}$, respectively. For seasoned mackerel, those corresponding contents were 8.5 and $8.6 \mathrm{mg} \mathrm{kg}^{-1}$, respectively. Ratios of data by HILIC-MS/MS against by HPLC-FL were from 96.1 to $98.8 \%$. The results determined using HILIC-MS/MS were correlated with those determined using HPLC-FL. 
These results show that HILIC-MS/MS is a reliable and rapid method for determining histamine in seafood samples.

The proposed HILIC-MS/MS method for seafood histamine was validated as mentioned above. Concretely, LOD was $0.015 \mathrm{mg} \mathrm{kg}^{-1}$, LOQ was $0.052 \mathrm{mg} \mathrm{kg}^{-1}$, and good linearity $\left(R^{2}=0.999\right)$ was obtained in the range from 0.4 to $200 \mathrm{mg} \mathrm{kg}^{-1}$ for $5 \mathrm{~g}$ of seafood samples. Good recoveries, repeatability and inter-day RSD were obtained, even while the method was much simpler than the conventional HPLC-FL method.

\section{Conclusions}

An analytical method for histamine, which is of most concern in chemical food poisoning cases in Japan, has been developed by means of HILIC-MS/MS using a sulfobetaine zwitterion column. Satisfactory results were obtained in recovery tests and in real seafood sample analysis. The proposed HILIC-MS/MS method does not require sample purification because of the high selectivity of LC-MS/MS; this dramatically simplifies the method. The pretreatment time for this method is $1 \mathrm{~h}$, which is a quarter of that required for the conventional HPLC-FL method. Furthermore, the new method uses less organic solvents and reagents. The new method is therefore useful for the rapid determination of the source of chemical food poisoning.

\section{References}

1. K. Kan, H. Ushiyama, T. Shindo, S. Uehara, and K. Yasuda, J. Food Hyg. Soc. Jpn., 2000, 41, 116.

2. M. Toda, M. Yamamoto, C. Uneyama, and K. Morikawa, Bull. Natl. Inst. Health Sci., 2009, 127, 31.

3. Pharmaceutical Society of Japan (ed.), "Eiseishikenhou, Chukai (Standard Methods of Analysis for Chemists, in Japanese)", 2010, Kaneharashuppan, Tokyo, 199.

4. K. Saito, M. Horie, Y. Tokumaru, S. Hattori, and H. Nakazawa, J. Food Hyg. Soc. Jpn., 1998, 39, 39.

5. M. Nakashima and A. Sugiyama, J. Food Hyg. Soc. Jpn., 1999, 40, 285.

6. A. J. Alpert, J. Chromatogr., 1990, 499, 177.

7. Y. Iwasaki, K. Inoue, R. Ito, Y. Yoshimura, K. Saito, and H. Nakazawa, Bunseki Kagaku, 2005, 54, 135.

8. T. Ikegami, H. Takubo, and N. Tanaka, Chromatogr., 2008, $29,1$.

9. C. Lamouroux, G. Foglia, and G. Le Rouzo, J. Chromatogr., A, 2011, 1218, 3022.

10. P. Jandera, Anal. Chim. Acta, 2011, 692, 1.

11. V. Gianotti, U. Chiuminatto, E. Mazzuccoa, F. Gosetti, M. Bottaro, P. Frascarolo, and M. C. Gennaro, J. Chromatogr., A, 2008, 1185, 296. 